\title{
Low-lying Dirac eigenmodes and monopoles in 3+1D compact QED
}

\author{
Toru T. Takahashi * \\ September 26, 2021
}

\begin{abstract}
We study the properties of low-lying Dirac modes in quenched compact QED at $\beta=1.01$, employing $12^{3} \times N_{t}\left(N_{t}=4,6,8,10,12\right)$ lattices and the overlap formalism for the fermion action. We pay attention to the spatial distributions of low-lying Dirac modes below and above the "phase transition temperature" $T_{c}$. Near-zero modes are found to have universal anti-correlations with monopole currents, and are found to lose their temporal structures above $T_{c}$ exhibiting stronger spatial localization properties. We also study the nearestneighbor level spacing distribution of Dirac eigenvalues and find a Wigner-Poisson transition.
\end{abstract}

\section{Introduction}

In the previous paper [1, the spatial correlations between monopoles [2] and low-lying Dirac eigenmodes (eigenfunctions of the Dirac operator) were studied in 4D quenched compact QED, and it was found that there exist universal anti-correlations between them below and above the critical coupling $\beta_{c}$. The clear anti-correlation between Dirac eigenfunctions and monopoles implies that the level dynamics of Dirac eigenvalues, which is responsible for the chiral phase transition via Banks-Casher relation 3 , is controlled by monopole configurations in $4 \mathrm{D}$ Euclidean system 1 . In the strong coupling phase $\left(\beta<\beta_{c}\right)$, monopoles form global and complicated clusters and make the vacuum complex bringing about repulsive forces among Dirac eigenvalues [1, 4, 5]. This repulsive force among eigenvalues, which is observed as the Wigner distribution in the neighboring level spacings, forms the non-vanishing spectral density at the spectral origin, which is equivalent to the non-vanishing chiral condensate $\langle\bar{\psi} \psi\rangle$. On the other hand, in the weak coupling phase $\left(\beta>\beta_{c}\right)$, large monopole clusters vanish and the vacuum structure is much simpler, which leads to weaker repulsive forces among eigenvalues [1, 4, 5] and results in the Poisson statistics in the level spacings. This weaker repulsive force is not so strong that Dirac eigenvalues can form non-zero spectral density at the origin, and the chiral condensate vanishes in this phase.

We in Ref. [1] varied the couplings around the critical coupling $\beta_{c}$ employing isotropic $4 \mathrm{D}$ systems with the total volumes fixed, and investigated the natures of low-lying Dirac modes. In this case, the chirally symmetric vacuum realized at $\beta>\beta_{c}$ is rather simple and perturbative. It is however known that the "finite temperature" transition still exhibits several nonperturbative features. For example, in the case of QCD, the formation of the stronglycoupled quark-gluon plasma phase (sQGP) 6, 7, 8, just above the transition temperature was recently suggested and attracting many interests. Nonperturbative aspects above the transition temperature $T_{c}$ can be also observed in the Wilson loops. Whereas temporal Wilson loops exhibit deconfinement feature above $T_{c}$, spatial Wilson loops still show an area law (i.e. temporally deconfined and spatially confined), which hints that the vacuum structure is not simple but still complicated even above $T_{c}$. The $(3+1) \mathrm{D}$ compact QED has similar properties, and the remaining nonperturbative structures can be also found in it. We investigate the properties of Dirac eigenmodes, eigenvalues, and monopoles in quenched $(3+1) \mathrm{D}$ compact QED, and see what happens.

\footnotetext{
*Yukawa Institute for Theoretical Physics, Kyoto university, Kitashirakawa-Oiwakecho, Sakyo, Kyoto 606-8502, Japan
} 
The organization of this paper is as follows: In Sec. 2. we briefly show our formalism. Several properties of low-lying Dirac modes and eigenvalues are clarified in Sec. 3, Sec. 4 is devoted to the discussion based on numerical results. We summarize the paper in Sec. 5 ,

\section{Formalism}

We adopt the Wilson gauge action at $\beta=1.01$ for gauge fields,

$$
S_{\mathrm{QED}}=\beta \sum_{x} \sum_{\mu, \nu}\left(1-\cos \theta_{\mu \nu}(x)\right),
$$

and employ the overlap-Dirac operator [9, 10, which is constructed as

$$
D \equiv \rho\left[1+\gamma_{5} \operatorname{sgn}\left(H_{W}\right)\right] \equiv \rho\left[1+\gamma_{5} \frac{H_{W}}{\sqrt{H_{W}^{2}}}\right],
$$

and realizes the exact chiral symmetry on a lattice [11, 12. Here, $H_{W} \equiv \gamma_{5}\left(D_{W}-\rho\right)$ is the hermitian Wilson-Dirac operator defined with the standard Wilson-Dirac operator $D_{W}$. The "negative mass" $\rho$ is chosen in the range of $0<\rho<2$, which we set 1.6 throughout this paper. We impose the periodic boundary conditions in all the spatial direction for the fermion fields, and the anti-periodic boundary condition is imposed on the temporal boundary. We compute lowest 50 eigenpairs at each "temperature", with 48 gauge configurations. All the eigenvalues $\lambda_{\text {lat }}$ of $D$, which lie on a circle with the radius of $\rho$ in a complex plain, are stereographically projected onto the imaginary axis via Möbius transformation [13],

$$
\lambda=\frac{\lambda_{\text {lat }}}{1-\lambda_{\text {lat }} / 2 \rho} .
$$

The spatial volumes are all fixed to $12^{3}$ and we adopt 5 different temporal lengths, $N_{t}=1 / T$ $=4,6,8,10$, and 12. The "finite temperature" phase transition in compact QED was extensively investigated [14] and the "transition temperature" at $\beta=1.01$ was found to lie around $N_{t} \sim 6$. (See also Ref. 15].) We expect that our setup can cover the systems below and above the transition temperature, though finite volume effects might not be negligible and the phase transition temperature would be slightly modified.

\section{Low-lying Dirac eigenvalues and corresponding modes}

In Ref. [1, we found anti-correlations between (near-zero) Dirac modes and monopoles, which indicates that near-zero modes are "scattered" by monopoles, and it was conjectured that monopoles as impurities for near-zero modes are responsible for the "complexity" of a vacuum and control the level dynamics of low-lying Dirac eigenvalues [1. Abundance of monopoles implies a complex vacuum and the level spacing distributions of Dirac eigenvalues obey the Wigner distribution. On the other hand, absence of monopoles makes a vacuum simple and the Poisson-like distribution appears. The Wigner (Poisson-like) distribution is the consequence of strong (weak) repulsive forces among Dirac eigenvalues, and strong (weak) repulsive forces are responsible for a non-vanishing (vanishing) spectral density at the spectral origin. The spectral density at the origin is finally related to chiral condensate $\langle\bar{\psi} \psi\rangle$ via the Banks-Casher relation [3]. Chiral condensate $\langle\bar{\psi} \psi\rangle$ simply reflects the complexity of a vacuum, which is brought about by monopoles' degrees of freedom. These results in Ref. 1] were all obtained in isotropic systems where the temporal length $N_{t}$ is equal to the spatial extent $N_{s}\left(N_{t}=N_{s} \gg 1 / T_{c}\right)$. Then, is this scenario the case also at "finite temperature" $\left(N_{t}<N_{s}\right)$ ? The answer would be no. Though the chirally symmetric vacuum in the weak coupling regime is actually simple [1, we expect that the symmetric vacuum realized at high temperature is still complex. In fact, monopole density is not much reduced even at high temperature (small $N_{t}$ ) region, which can be found in Fig. 1.

We go further with the clarification of the chiral phase transition in compact QED. The keywords are (1) spectral densities at the spectral origin, (2) level spacing distributions of Dirac eigenvalues, (3) correlations between low-lying Dirac modes and monopoles, and (4) spatial distributions of low-lying Dirac modes. 


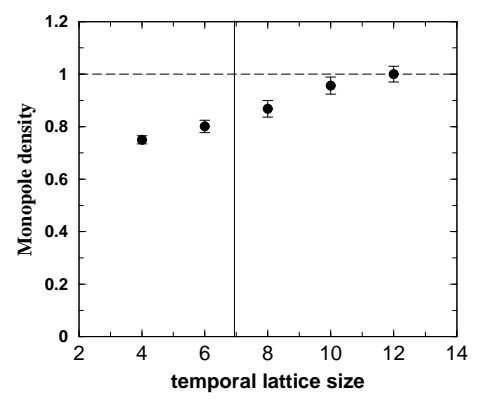

Figure 1: The normalized monopole densities (total monopole lengths divided by total volumes) are plotted. The solid line is drawn at $N_{t} \sim 7$ for reference.

\begin{tabular}{c|ccccc}
\hline \hline$N_{t}$ & 4 & 6 & 8 & 10 & 12 \\
\hline$\nu=0$ & 41 & 38 & 41 & 28 & 32 \\
$\nu=1$ & 6 & 10 & 7 & 17 & 12 \\
$\nu=2$ & 1 & 0 & 0 & 3 & 4 \\
\hline \hline
\end{tabular}

Table 1: The numbers of exact zero-modes found in 48 gauge configurations at each inverse temperature $N_{t}$ are listed. The $i$-th row gives the number of configurations with $0,1,2$ zeromode(s), respectively.

\subsection{Spectral densities and level spacing distributions}

We show in Fig. 2 the histograms of Dirac eigenvalues at $N_{t}=12,8$, and 4 . The numbers of
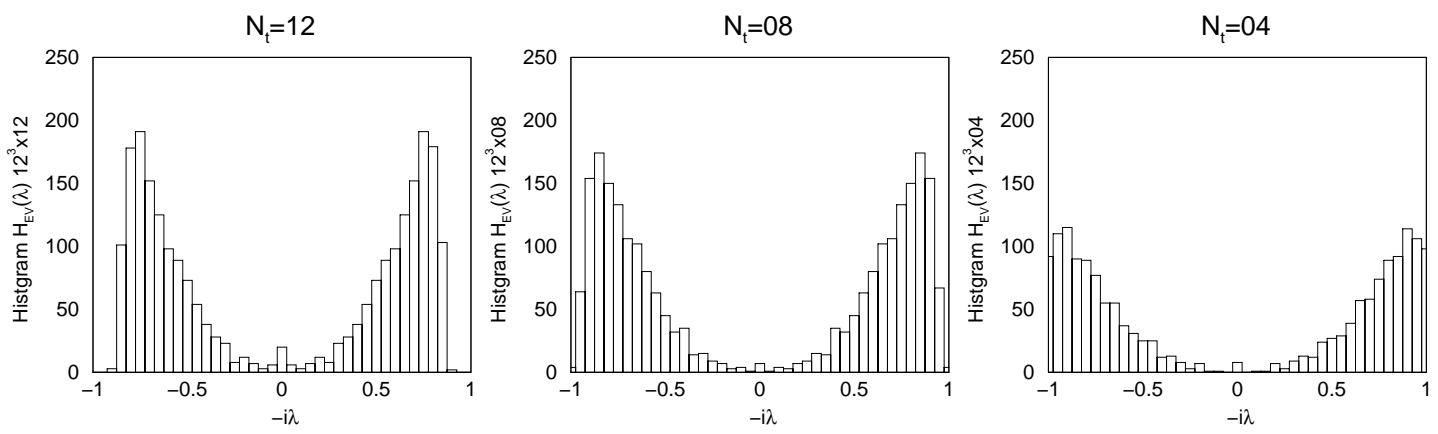

Figure 2: The histograms $H_{\mathrm{ev}}(\lambda)$ of the eigenvalues $\lambda$ of the overlap-Dirac operator $D$ are plotted for each $N_{t}(12,8,4)$. The horizontal axis denotes $-i \lambda$. All the eigenvalues $\lambda_{\text {lat }}$ lying on a circle in a complex plain are stereographically projected onto the imaginary axis via Möbius transformation, $\lambda=\frac{\lambda_{\text {lat }}}{1-\lambda_{\text {lat }} / 2 \rho}$.

zero modes found in 48 gauge configurations at each inverse temperature $1 / T=N_{t}(4,6,8$, $10,12)$ are listed in Table1. The histograms around the spectral origin at $N_{t}=8,12$ are found to be rather flattened and have non-zero heights, while that at $N_{t}=4$ exhibits the vanishing density. These densities are related to the non-vanishing (vanishing) chiral condensate at $N_{t}>6 \sim 1 / T_{c}\left(N_{t}<6 \sim 1 / T_{c}\right)$ via the Banks-Casher relation. It is remarkable that the chiral phase transition is almost accompanied by the deconfinement transition, which would occur around $N_{t} \sim 6$. The vanishing density seen in the $12^{3} \times 4$ system could be the consequence of some finite (spatial) volume effects. In order to ensure that it is not the case, we show the normalized histograms $H_{\mathrm{ev}}(\lambda)$ obtained with $12^{3} \times 4,14^{3} \times 4$, and $16^{3} \times 4$ lattices in Fig. [3]left]. All three histograms exhibit the same behavior, and we can neglect 

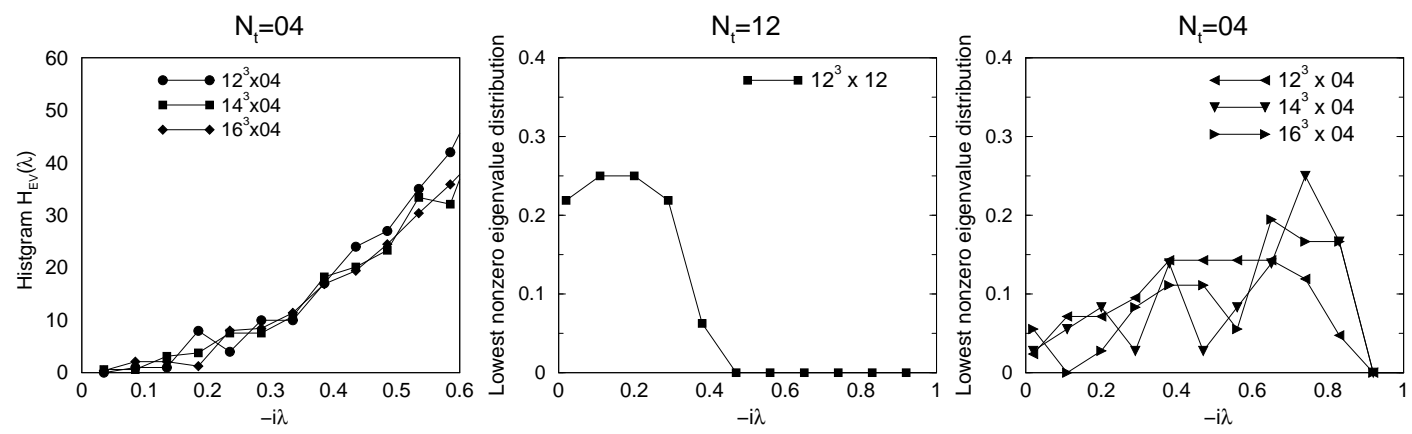

Figure 3: Left: The histograms $H_{\mathrm{ev}}(\lambda)$ of the eigenvalues $\lambda$ of the overlap-Dirac operator $D$, which are obtained with $12^{3} \times 4,14^{3} \times 4$, and $16^{3} \times 4$ lattices. Middle and Right: The distributions of lowest nonzero eigenvalues at $N_{t}=12$ and 4 obtained with $\nu=0$ gauge configurations.

the finite volume effects in $12^{3} \times 4$ system. The lowest-nonzero-eigenvalue distributions at $N_{t}=12$ and 4 in $\nu=0$ sector are respectively plotted in Fig. [3 [middle] and Fig. [3] right], for the purpose of reference.

As actually demonstrated in Refs. [1, 16, the spectral density at the spectral origin is enhanced by the "repulsive force" among eigenvalues, and this force can be clarified with the neighboring level spacing distributions of Dirac eigenvalues. We show in Fig. 4 the unfolded neighboring level spacing distributions $P_{\text {lat }}(s)$ obtained at $N_{t}=12,8$, and 4 . The solid lines denote the Wigner distribution function $P_{\mathrm{Wig}}(s) \equiv \frac{32}{\pi^{2}} s^{2} \exp \left(-\frac{4}{\pi} s^{2}\right)$, which is a good approximation of the original distribution obtained by the random matrix theory with chiral unitary ensemble, and the dashed lines the Poisson distribution function $P_{\mathrm{Poi}}(s)=$ $\exp (-s)$. We again find the Wigner-Poisson transition, which was also found in Ref. [1] and is consistent with the spectral densities at the origin.
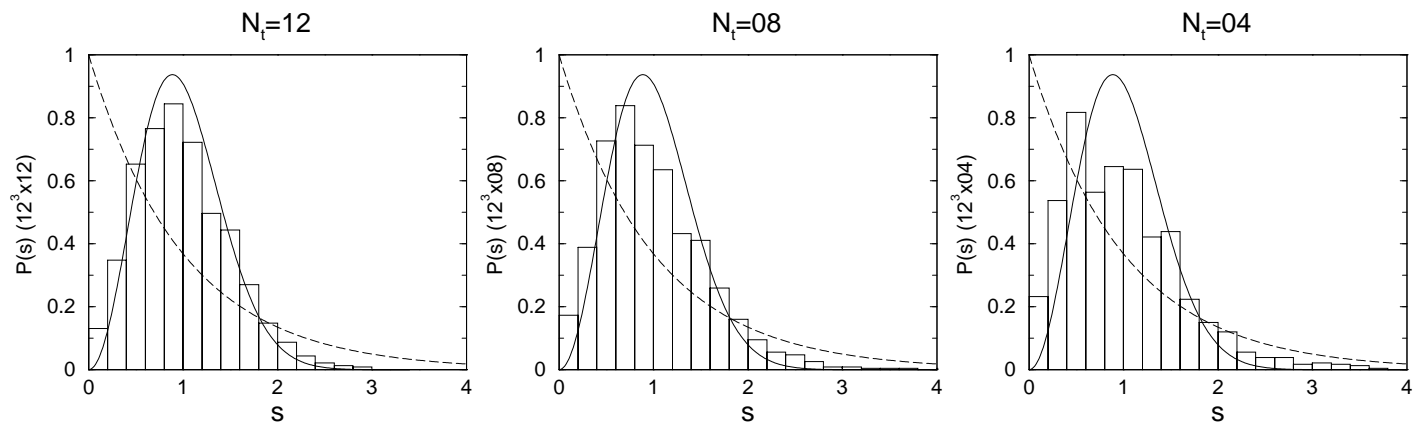

Figure 4: The unfolded nearest-neighbor level spacing distributions $P_{\text {lat }}(s)$ at $N_{t}=12,8$, and 4. The solid lines denote the Wigner distribution function $P_{\mathrm{Wig}}(s) \equiv \frac{32}{\pi^{2}} s^{2} \exp \left(-\frac{4}{\pi} s^{2}\right)$ and the dashed lines the Poisson distribution function $P_{\text {Poi }}(s)=\exp (-s)$.

\section{2 low-lying Dirac modes and monopoles}

We next investigate the correlations between low-lying modes and monopoles following Ref. [1. For this aim, we define and investigate the histogram ratios $R_{\psi}\left(\rho_{\psi}\right)$ [1] defined as

$$
R_{\psi}\left(\rho_{\psi}\right) \equiv \frac{H_{\psi}^{\text {mon }}\left(\rho_{\psi}\right)}{H_{\psi}^{\text {all }}\left(\rho_{\psi}\right)}
$$


The eigenmode density $\rho_{\psi} \equiv \sum_{\alpha}\left|\psi_{\lambda}(x)\right|^{2}$ here is the absolute squares of an eigenfunction $\psi_{\lambda}(x)$ locally summed up over the spinor index. $H_{\psi}^{\text {all }}\left(\rho_{\psi}\right)\left(H_{\psi}^{\operatorname{mon}}\left(\rho_{\psi}\right)\right)$ denotes the histogram of $\rho_{\psi}$ evaluated at all the sites (only on monopoles). This quantity $R_{\psi}\left(\rho_{\psi}\right)$ equals to 1 , if there is no correlation between the spatial fluctuations of Dirac modes and monopoles. In the case when a positive (negative) correlation exists between the spatial fluctuations of Dirac modes and monopoles, $R_{\psi}\left(\rho_{\psi}\right)>1$ at smaller (larger) $\rho_{\psi}$ and $R_{\psi}\left(\rho_{\psi}\right)<1$ at large (smaller) $\rho_{\psi}$ hold. We show in Fig. 5 the histogram ratios for near-zero modes obtained at $N_{t}=12,8$, and 4. Display ranges are chosen as $0 \leq \rho_{\psi} \leq 0.0002 \times \frac{12}{N_{t}}$ with intrinsic densities in mind. The universal anti-correlations are again observed. (We here omit the results on exact-zero modes 17, 18, since they show no apparent correlation with monopoles. This tendency was also found in our previous paper [1. The present strategy is rather simple and we would need more sophisticated analyses to cast light on the properties of exact-zero modes. In the following sections, attentions will be paid mainly to near-zero Dirac modes.)
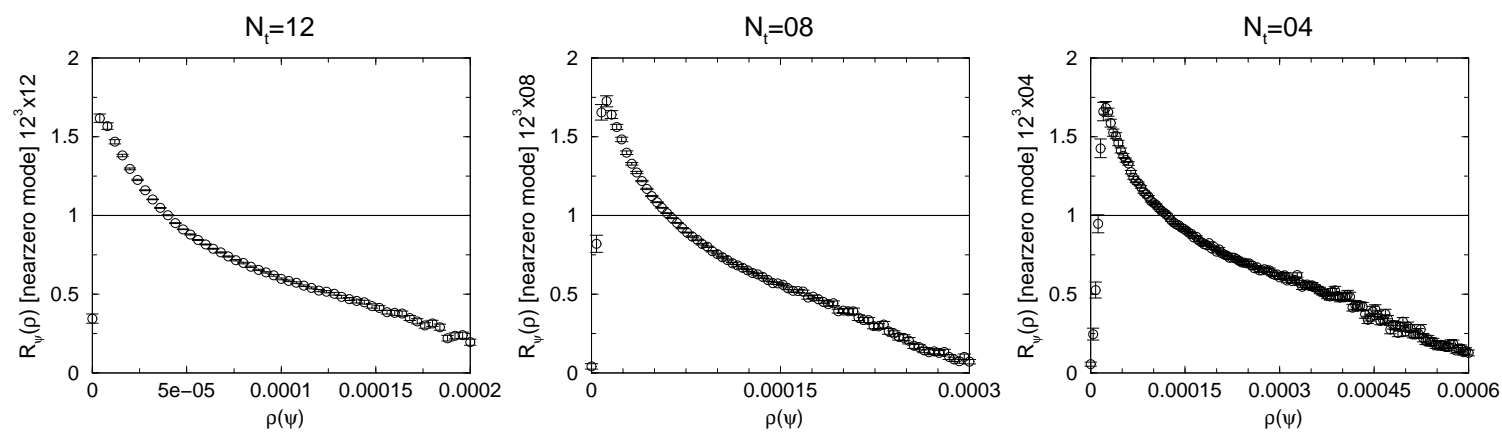

Figure 5: The histogram ratios $R_{\psi}\left(\rho_{\psi}\right)$ for near-zero modes at $N_{t}=12,8$, and 4 are plotted in the range of $0 \leq \rho_{\psi} \leq 0.0002 \times \frac{12}{N_{t}}$. We draw a line at $R_{\psi}\left(\rho_{\psi}\right)=1$ for reference.

\subsection{Spatial distributions of low-lying Dirac modes}

To clarify the spatial distributions of Dirac eigenmodes, it is convenient to extract the inverse participation ratio (IPR) of each eigenmode. The eigenmode's distribution is partially encoded in the corresponding IPR: The IPR is unity when $\psi_{\lambda}(x)$ maximally spreads over the system, and equals to $V$ in the case when $\psi_{\lambda}(x)$ lives only on a single site, reflecting the spatial distribution of the eigenfunction $\psi_{\lambda}(x)$.

The IPR $I(\lambda)$ of $\psi_{\lambda}(x)$ is defined as

$$
I(\lambda)=V \sum_{x} \rho_{\mathrm{IPA}}(x)^{2}, \quad \rho_{\mathrm{IPR}}(x) \equiv \sum_{\alpha}\left|\psi_{\lambda}(x)\right|^{2} .
$$

Here, $V$ denotes the system volume and $\psi_{\lambda}(x)$ is the eigenfunction associated with an eigenvalue $\lambda$ normalized as $\sum_{x}\left|\psi_{\lambda}(x)\right|^{2}=1$. The Greek alphabet $\alpha$ is the index for a spinor. The density $\rho_{\mathrm{IPR}}(x)$ is obtained by locally summing up the absolute square of each component of an eigenfunction $\psi_{\lambda}(x)$ only over its spinor index.

We show the scatter plots of the IPRs of low-lying modes in Fig. 6. The IPRs of near-zero modes approximately range from 1 to 2 at $N_{t}=8$ and 12 . On the other hand, at $N_{t}=4$, many of the IPRs of near-zero modes take much larger values indicating the strong localizations of near-zero modes above $T_{c}$. Near-zero modes are localized and the overlaps among them get much smaller, which would be the source of the Poisson-like distribution in the neighboring level spacings of Dirac eigenvalues: Level repulsions would not occur without overlaps among eigenfunctions. Weaker repulsive forces among eigenvalues characterized by such a Poissonlike statistics lead to the vanishing eigenvalue density at the spectral origin, and the chiral symmetry is restored. The chiral phase transition across $T_{c}$ is now found to be essentially different from that across $\beta_{c}$ [1]. The Poisson-like statistics above $\beta_{c}$, where near-zero Dirac modes are completely delocalized and are plane-wave like, is caused by the "simpleness" of 
the vacuum. That above $T_{c}$ is however driven by the localization of near-zero modes. We expect nontrivial vacuum structures above $T_{c}$.
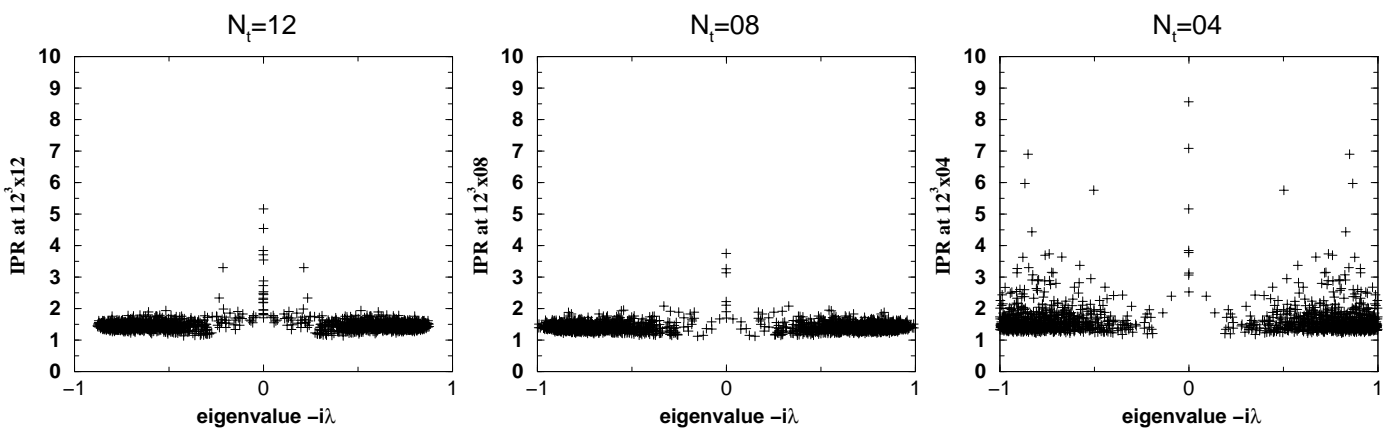

Figure 6: The scatter plots of inverse participation ratios of low-lying Dirac modes at $N_{t}=12$, 8 , and 4 . The horizontal axis denotes $-i \lambda$, the associated eigenvalue to each Dirac mode.

We define another type of IPR $I s(\lambda ; \mu)$, which is nothing but the IPR evaluated in a 3 -dim subspace at $x_{\mu}=$ const., for further clarification of Dirac modes' distributions above $T_{c}$. The subspace-IPR $I s(\lambda ; \mu)$ is defined as,

$$
I s(\lambda ; \mu) \equiv V_{s} \sum_{x_{\mu}=\text { const. }} \rho_{\mathrm{IPA}}^{\prime}(x ; \mu)^{2}, \quad \rho_{\mathrm{IPR}}^{\prime}(x ; \mu) \equiv \sum_{\alpha}\left|\psi_{\lambda}(x)\right|^{2} / \sum_{\alpha, x, x_{\mu}=\text { const. }}\left|\psi_{\lambda}(x)\right|^{2} .
$$

Here, $V_{s}$ denotes the volume of the 3 -dim subspace. We evaluate the ratios, $I s(\lambda ; i) / I(\lambda)(i=$ $1,2,3)$ and $I s(\lambda ; 4) / I(\lambda)$. In case an eigenfunction is simply extended along the temporal direction and spatially localized, $I s(\lambda ; 4) / I(\lambda)$ is just unity and $I s(\lambda ; i) / I(\lambda)(i=1,2,3)$ is expected to be less than 1 , because in this case the eigenfunction has no structure along the time-direction and only the spatial structure is responsible for the total IPR. We show in Fig. $7 \mathrm{I} I s(\lambda ; 4) / I(\lambda)$ and $I s(\lambda ; i) / I(\lambda)(i=1,2,3)$ obtained in $12^{3} \times 4$ lattice. One can find that $I s(\lambda ; 4) / I(\lambda) \simeq 1$ and $I s(\lambda ; i) / I(\lambda)(i=1,2,3) \ll 1$ hold, which indicates the vanishing temporal structure of low-lying Dirac modes at $N_{t}=4$. All the ratios, $I s(\lambda ; 4) / I(\lambda)$ and $I s(\lambda ; i) / I(\lambda)(i=1,2,3)$, evaluated at $4 \leq N_{t} \leq 12$ are shown in Fig. 8 , The temporal structures of low-lying modes quickly vanish around $N_{t} \sim 6 \sim 1 / T c$.
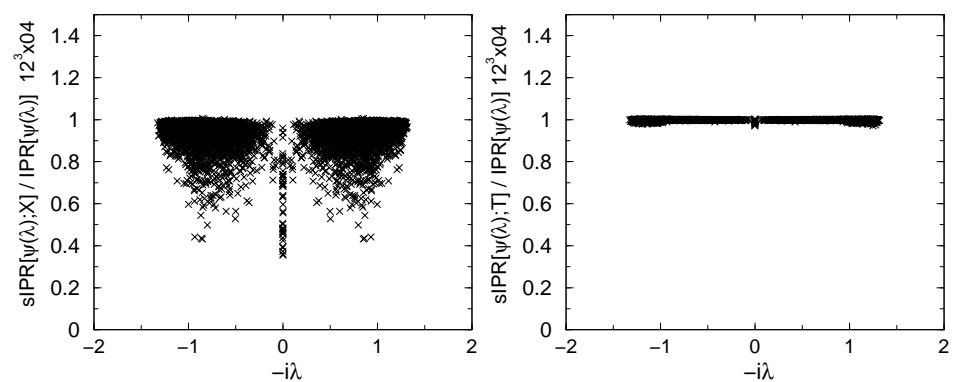

Figure 7: The IPR-ratios, $I s(\lambda ; i) / I(\lambda)(i=1,2,3)$ [left] and $I s(\lambda ; 4) / I(\lambda)$ [right] obtained with the $12^{3} \times 4$ lattice are plotted.

\section{Discussions}

Now that we have found that, at "high temperature", low-lying (near-zero) Dirac modes lose their temporal structures and are spatially localized. We have two questions: (1) Why do the temporal structures of near-zero modes vanish? (2)Why are near-zero modes spatially (and 

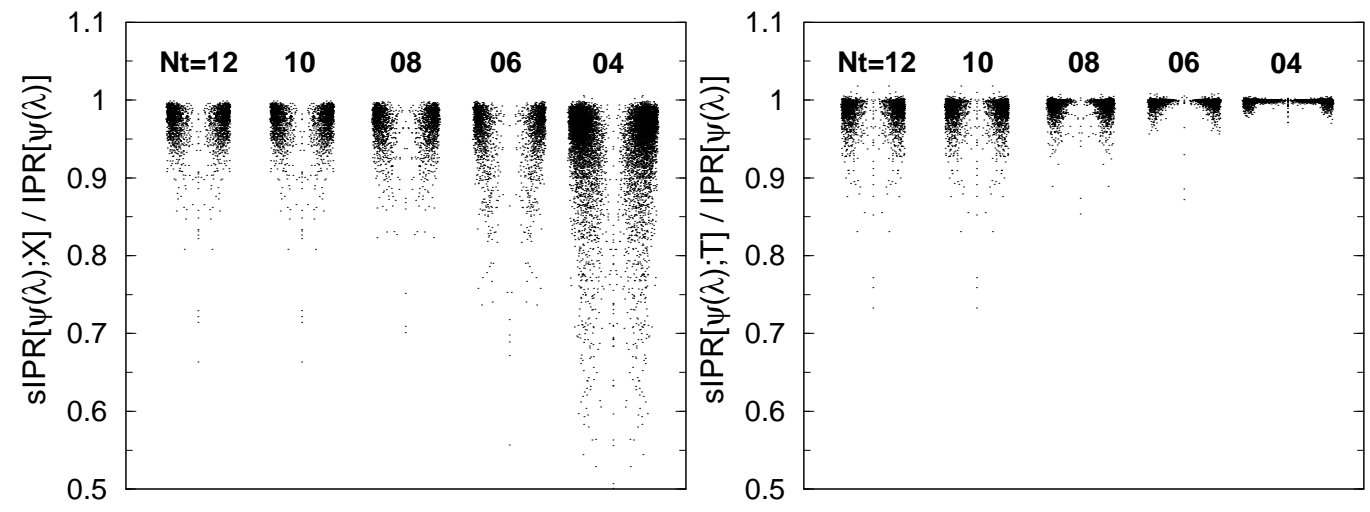

Figure 8: The IPR-ratios, $I s(\lambda ; i) / I(\lambda)(i=1,2,3)$ [left] and $I s(\lambda ; 4) / I(\lambda)$ [right] obtained with $12^{3} \times 4 \sim 12^{3} \times 12$ lattice are plotted.

strongly) localized? As we have just found or as can be found in Ref. 1], near-zero Dirac modes have anti-correlations with monopoles and then monopoles are surely responsible for the distributions of near-zero modes. These features may come from the metamorphosis of monopole world lines.

Monopole world lines will take "static" configurations at high temperature; most of the world lines are twisted around the 4-dim torus along the temporal direction as is illustrated in Fig. 9]left]. In such a case, near-zero modes can be freely extended along the temporal direction, since they do not encounter monopoles as impurities for near-zero modes, which is schematically illustrated in Fig. 9 middle]. This "clearing up" along the temporal direction may be the main cause for the vanishing temporal structures of near-zero modes. It could be also the origin of the stronger localization properties of near-zero modes: The clearing up leads to the irrelevance of the temporal direction and implies that the system dimensionality for near-zero Dirac modes is reduced approximately to three. The near-zero modes are then expected to get strongly localized due to this dimensional reduction. (Dimensionality also plays an essential role in the localization of wavefunctions. It is a celebrated fact that, in a system whose dimensionality is lower than two, any random potentials readily lead to the exponential localization of wavefunctions.)

One may wonder which is the main origin of the effective dimensional reduction, clearing up along the temporal direction or shorter temporal extent $N_{t}$ ("thermal" effect). To answer this question, we perform exactly the same analyses in an artificially constructed isotropic $12^{3} \times 12$ system. We define link variables $U_{\mu}^{(2)}(\mathbf{x}, t)$ for this new system with $U_{\mu}(\mathbf{x}, t)$ in the original $12^{3} \times 4$ lattice. $U_{\mu}^{(2)}(\mathbf{x}, t)$ is defined as

$$
U_{\mu}^{(2)}(\mathbf{x}, t) \equiv U_{\mu}(\mathbf{x}, t \bmod 4) \quad(0 \leq t \leq 11) .
$$

In other words, we construct an isotropic $12^{3} \times 12$ system by piling up three $12^{3} \times 4$ systems. (See Fig. 9 right].) Due to the periodicity of U(1) gauge fields, monopole configurations are the same in these two systems. Though this newly constructed lattice is an isotropic system, monopole world lines exhibit static configurations. We show in Fig. 10 the histogram ratio $R_{\psi}\left(\rho_{\psi}\right)$ for near-zero modes, the scatter plot of inverse participation ratios of low-lying Dirac modes, the unfolded nearest-neighbor level spacing distributions $P_{\text {lat }}(s)$, the IPR-ratio $I s(\lambda ; i) / I(\lambda)(i=1,2,3)$, and the IPR-ratio $I s(\lambda ; 4) / I(\lambda)$. Surprisingly, all the quantities essentially remain the same, which indicates that the properties of low-lying modes do not depend on the temporal lattice extent at all, and that the temporal dimension is actually irrelevant.

The chiral phase transition in compact QED system is controlled by the metamorphosis of monopole world lines: When a system is covered by large monopole clusters, the system is complex [1] and the spectra of Dirac operators exhibit the Wigner distribution, which implies strong repulsions among Dirac eigenvalues and gives rise to the non-vanishing spectral density 

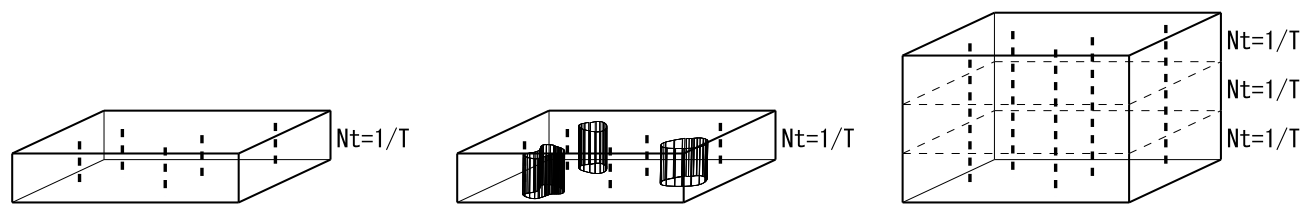

Figure 9: Left: Monopoles take rather static configurations with small $N_{t}$ (at high temperature). Middle: Near-zero Dirac modes are localized avoiding monopoles. In the presence of static monopoles, near-zero modes would be spatially localized and temporally delocalized. Right: We artificially construct $12^{3} \times 12$ system duplicating $12^{3} \times 4$ system. Due to the periodicity of gauge fields, monopole configurations on this artificial $12^{3} \times 12$ system are the same as those in the original $12^{3} \times 4$ system.

at the origin $(\langle\bar{\psi} \psi\rangle \neq 0)$. On the other hand, at high temperature, monopole world lines take static configurations and the system undergoes the clearing up along the temporal direction, which would lead to the effective dimensional reduction for near-zero modes and to the exponential localization of the modes. The exponential localization of Dirac modes causes weaker repulsive forces among eigenvalues exhibiting the Poisson-like distribution of Dirac spectra. Such weak repulsive forces cannot form non-zero spectral density at the spectral origin $(\langle\bar{\psi} \psi\rangle=0)$. The metamorphosis of monopole world lines is also responsible for the deconfinement phase transition. In a system where large monopole clusters cover the entire volume, it is in confinement phase. When monopole world lines take static configurations, the system breaks out from the confinement phase to the deconfinement phase. Such static monopoles still lead to the spatial confinement, which is the remnant of the confinement and gives rise to remaining nonperturbative phenomena.

These two phase transitions are then considered to be both controlled by the monopole dynamics. The coincidence of two "transition temperatures" could be naturally understood from this viewpoint. At least, if the metamorphosis of monopole world lines in the vicinity of the phase transition point is quick enough, the transition temperatures would be similar in magnitude. The clarification of Polyakov loops in terms of Dirac spectra [19] could also give us deeper understanding of the possible relationship between confinement and chiral symmetry breaking.

\section{Summary}

We have studied the properties of low-lying Dirac modes in quenched compact QED at $\beta=1.01$, employing $12^{3} \times N_{t}$ lattices with $N_{t}=1 / T=4,6,8,10,12$. The overlap formalism has been adopted for the fermion action.

We have found several features worth noting:

- The nearest-neighbor level spacing distribution of Dirac eigenvalues coincides with the Wigner distribution at $N_{t}=12$ (confinement phase), and it gradually changes to the Poisson distribution as $N_{t}$ is decreased, which is consistent with the spectral density at the spectral origin.

- Near-zero modes exhibits stronger localization at high temperature, which is the origin of the Poisson-like distribution of Dirac spectra.

- Near-zero modes lose their temporal structures above $T_{c}$.

- Near-zero modes have been found to have universal anti-correlations again with monopole world lines below and above the critical temperature.

The chiral phase transition at "high temperature" in compact QED seems to be controlled by the metamorphosis of monopole world lines. Monopole world lines take static configurations at high-T system and the system undergoes the clearing up along the temporal direction, which would lead to the effective dimensional reduction for near-zero modes. This reduction is responsible for the exponential localization of the modes, which causes weaker repulsive 

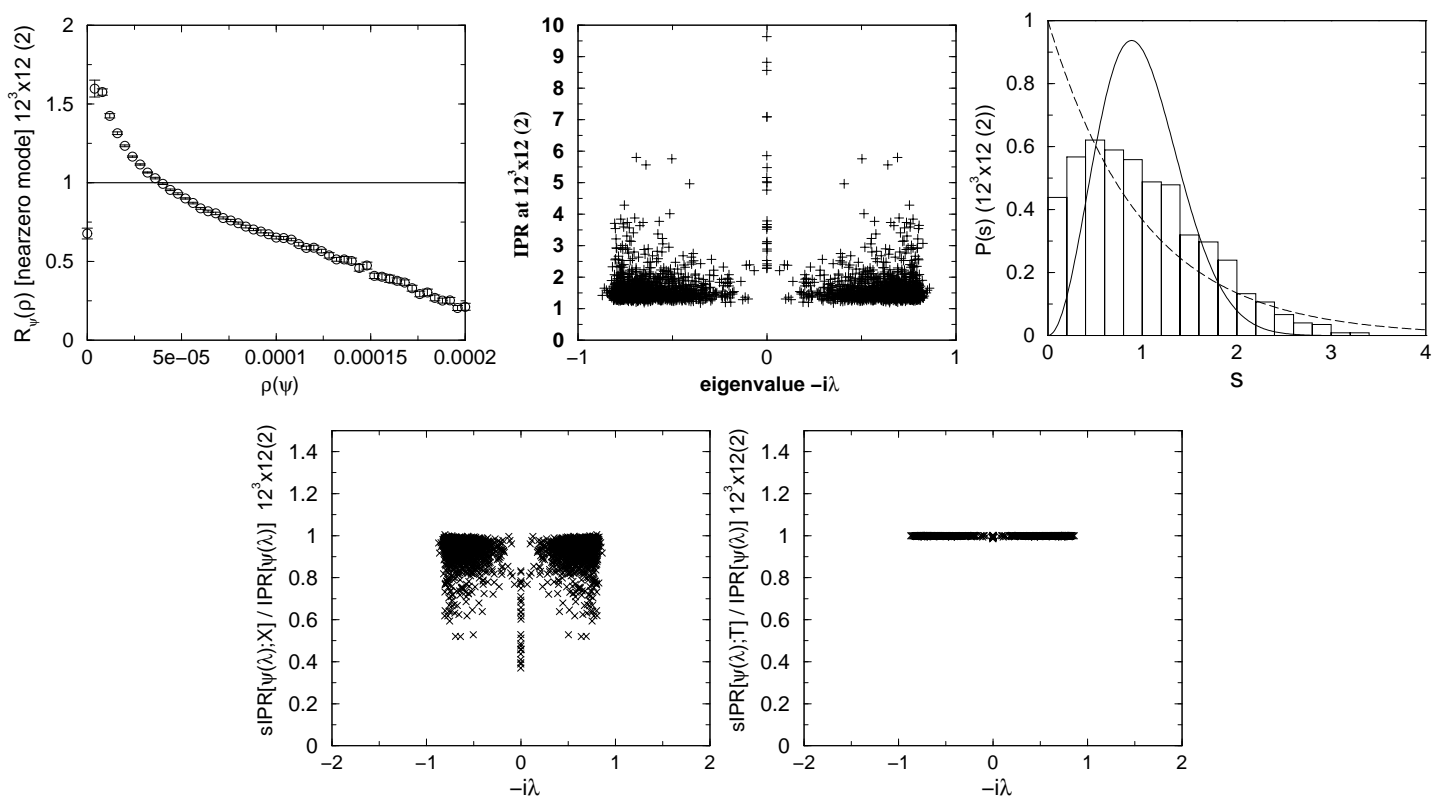

Figure 10: Several quantities obtained with the artificially constructed $12^{3} \times 12$ lattice are plotted; upper left: The histogram ratio $R_{\psi}\left(\rho_{\psi}\right)$ for near-zero modes, upper middle: the scatter plot of inverse participation ratios of low-lying Dirac modes, upper right: the unfolded nearest-neighbor level spacing distributions $P_{\text {lat }}(s)$, lower left: the IPR-ratio $I s(\lambda ; i) / I(\lambda)(i=$ $1,2,3)$, lower right: the IPR-ratio $I s(\lambda ; 4) / I(\lambda)$. Surprisingly, all these results remain almost the same as those in $12^{3} \times 4$ system.

forces among eigenvalues and gives rise to the Poisson-like distribution of Dirac spectra. Due to such weak repulsive forces, the spectral density at the spectral origin vanishes $(\langle\bar{\psi} \psi\rangle=0)$. Taking into account that the metamorphosis of monopole world lines is also responsible for the deconfinement transition, we conjecture from the microscopic viewpoint that the chiral and confinement/deconfinement transitions in compact QED are both induced by a single origin, monopole's dynamics.

\section{acknowledgments}

The author thanks Dr. K. Fukushima for useful comments. All the numerical calculations in this paper were carried out on NEC SX-8 at the Yukawa Institute Computer Facility and on NEC SX-8R at CMC and RCNP, Osaka university. This work was supported by the 21st Century COE "Center for Diversity and University in Physics", Kyoto University and Yukawa International Program for Quark-Hadron Sciences (YIPQS).

\section{References}

[1] T. T. Takahashi, JHEP 0711, 047 (2007) arXiv:hep-lat/0703023.

[2] T. A. DeGrand and D. Toussaint, Phys. Rev. D 22, 2478 (1980).

[3] T. Banks and A. Casher, Nucl. Phys. B 169, 103 (1980).

[4] M. V. Berry and M. Tabor, J. Phys. A 10 (1977) 371.

[5] O. Bohigas, M. J. Giannoni and C. Schmit, Phys. Rev. Lett. 52, 1 (1984).

[6] E. Shuryak, Prog. Part. Nucl. Phys. 53, 273 (2004) arXiv:hep-ph/0312227.

[7] T. Umeda, K. Nomura and H. Matsufuru, Eur. Phys. J. C 39S1 (2005) 9 arXiv:hep-lat/0211003. 
[8] M. Asakawa and T. Hatsuda, Phys. Rev. Lett. 92, 012001 (2004) arXiv:hep-lat/0308034.

[9] H. Neuberger, Phys. Lett. B 417, 141 (1998) arXiv:hep-lat/9707022.

[10] H. Neuberger, Phys. Lett. B 427, 353 (1998) arXiv:hep-lat/9801031.

[11] M. Luscher, Phys. Lett. B 428, 342 (1998) [arXiv:hep-lat/9802011.

[12] P. H. Ginsparg and K. G. Wilson, Phys. Rev. D 25, 2649 (1982).

[13] F. Farchioni, arXiv:hep-lat/9902029

[14] M. Vettorazzo and P. de Forcrand, Phys. Lett. B 604, 82 (2004) arXiv:hep-lat/0409135.

[15] M. Panero, JHEP 0505, 066 (2005) arXiv:hep-lat/0503024.

[16] A. M. Garcia-Garcia and J. C. Osborn, Phys. Rev. D 75, 034503 (2007) arXiv:hep-lat/0611019.

[17] B. A. Berg, U. M. Heller, H. Markum, R. Pullirsch and W. Sakuler, Phys. Lett. B 514, 97 (2001) arXiv:hep-lat/0103022.

[18] T. Drescher and C. B. Lang, JHEP 0502, 058 (2005) arXiv:hep-lat/0411012.

[19] C. Gattringer, Phys. Rev. Lett. 97, 032003 (2006) arXiv:hep-lat/0605018. 